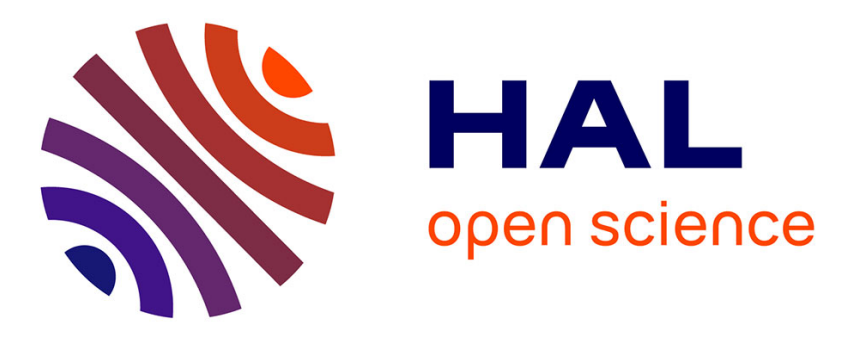

\title{
Dual-fibre stretcher and coma as tools for independent 2nd and 3rd order tunable dispersion compensation in a fibre-based 'scan-free' time domain optical coherence tomography system
}

L. Froehly, S. Iyer, F. Vanholsbeeck

\section{To cite this version:}

L. Froehly, S. Iyer, F. Vanholsbeeck. Dual-fibre stretcher and coma as tools for independent 2nd and 3rd order tunable dispersion compensation in a fibre-based 'scan-free' time domain optical coherence tomography system. Optics Communications, 2011, 284 (16-17), pp.4099-4106. 10.1016/j.optcom.2011.04.034 . hal-00611053

\section{HAL Id: hal-00611053 \\ https://hal.science/hal-00611053}

Submitted on 22 Apr 2021

HAL is a multi-disciplinary open access archive for the deposit and dissemination of scientific research documents, whether they are published or not. The documents may come from teaching and research institutions in France or abroad, or from public or private research centers.
L'archive ouverte pluridisciplinaire HAL, est destinée au dépôt et à la diffusion de documents scientifiques de niveau recherche, publiés ou non, émanant des établissements d'enseignement et de recherche français ou étrangers, des laboratoires publics ou privés.

\section{(ㄷ)(i)}

Distributed under a Creative Commons Attribution| 4.0 International License 


\title{
Dual-fibre stretcher and coma as tools for independent 2nd and 3rd order tunable dispersion compensation in a fibre-based 'scan-free' time domain optical coherence tomography system
}

\author{
Luc Froehly $^{\mathrm{a}, *}$, Sairam Iyer ${ }^{\mathrm{b}}$, Frédérique Vanholsbeeck ${ }^{\mathrm{b}}$ \\ a Institut FEMTO-ST, UMR CNRS 6174, Université de Franche-Comté, France \\ b Department of Physics, The University of Auckland, New Zealand
}

Dispersion compensation up to the third order is experimentally demonstrated by using a dual-fibre stretcher combined with the coma of an imaging lens, in a fibre-based scan-free time domain optical coherence tomography system, leading to an axial resolution of less than $3 \mu \mathrm{m}$.

\section{Introduction}

Optical Coherence Tomography (OCT) is a non-invasive imaging technique that has attracted immense interest over the last decade, especially in the field of biomedical imaging [1]. Amongst the many configurations that can be found in literature, fibre-based systems present the distinct advantage of compactness and portability. However, the use of optical fibres often introduces a strong chromatic dispersion mismatch between the two arms of the interferometer, which if not properly compensated, leads to a loss of depth resolution [2]. Since optical sources with broad bandwidths are often utilised, accurate dispersion compensation becomes crucial [3]. In general, dispersion compensation in OCT is addressed through numerous techniques ranging from optical (prisms, rapid scanning optical delay line , acousto-optic modulators) to numerical compensation methods [4-7]. Dispersion compensation in fibre-based systems can in principle be obtained by finely adjusting the fibre lengths (to sub$\mathrm{mm}$ precision), but this is very impractical. Recently, Iyer et al. showed that dispersion compensation with a dual-fibre stretcher system allows for an all-fibre fine tuning of the dispersion imbalance by altering the differential dispersion of two different types of optical fibres [8]. This technique is however limited to the 2 nd order term and the remaining 3rd order dispersion cannot be properly compensated by using only the two stretchers. Recently, Froehly et al. showed that this 3rd order term can potentially be compensated by using the

\footnotetext{
* Corresponding author.

E-mail address: luc.froehly@univ-fcomte.fr (L. Froehly).
}

properties of diffraction gratings in a scan-free grating-based time domain OCT system [9]. In this paper, we show that by combining the two setups we are able to independently compensate for a variable amount of the 2 nd and 3rd order chromatic dispersion terms, given a source bandwidth of $165 \mathrm{~nm}$, which is equivalent to a depth resolution of less than $3 \mu \mathrm{m}$.

\section{Principle}

The setup that we use in this paper is a combination of two different configurations (labeled A and B in Fig. 1) which were published previously $[8,9]$. The former configuration is the fibre based system consisting of two fibre stretchers, while the latter is the scanfree time domain correlator. Both of these setups will be elaborated in Sections 2.1 and 2.2, respectively. Further, in Section 2.3 we describe the theory which allows us to compensate for the 3rd order dispersion term.

\subsection{Stretcher compensation}

The details of the fibre-based tunable dispersion compensator is described in [8]. In the system shown in Fig. 1(A), a fibre stretcher is present in both arms of the interferometer. Each stretcher is made up of different fibre types and is operated in parallel by elastically stretching or unstretching the fibres by the same length $\Delta L$. This allows us to keep the relative group delay constant, while adjusting the second order dispersion term independently, in each arm. Therefore, by operating the two stretchers in parallel, this system can compensate for a variable amount of chromatic dispersion with a strong second 


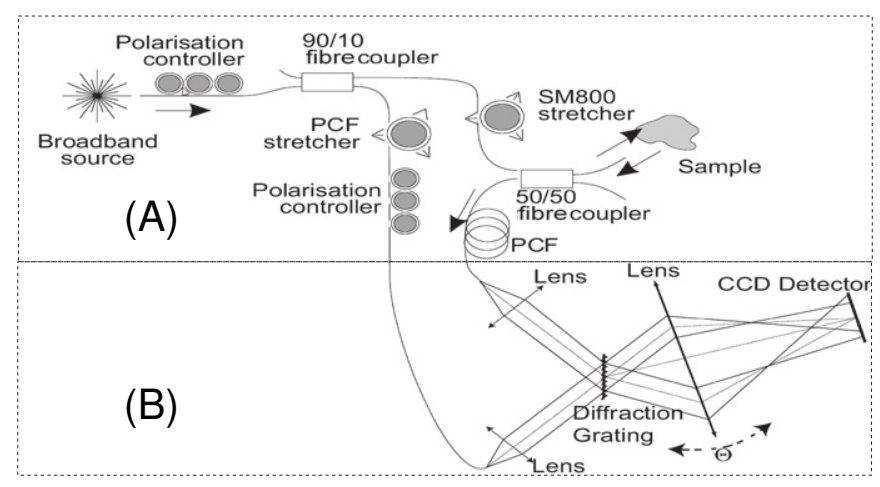

Fig. 1. Hybrid OCT setup combining two configurations: (A) fibre based interferometer with dual-fibre stretcher, (B) scan-free time domain correlator.

order coefficient, while simultaneously allowing adjustment of the group delay between the two arms of the interferometer.

\subsection{Scan-free time domain correlator}

Although this technique is still unfamiliar in the field of OCT, scanfree optical time domain correlations allow direct registration of the temporal correlation features without any temporal scanning or inverse Fourier transform. Such techniques originate from spectroscopy [10] and are widely used in ultra-short pulse shape measurement [11]. The application of scan-free time domain correlators to imaging has been the subject of numerous works, with each time different improvements and modalities; to have a complete overview of the actual state of the art the reader may refer to the review paper by Froehly and Leitgeb [12]. Scan free systems are inherently fast, but with a sensitivity almost $20 \mathrm{~dB}$ lower than that of FDOCT $[13,14]$. Despite this sensitivity decay, imaging capacity of such a system has been demonstrated and the actual state of the art with this technique shows performances good enough (93 dB sensitivity, $47 \mathrm{kHz}$ A-scan) to reach in-vivo imaging of biological tissues [15]. Such systems present interesting intrinsic properties such as, the ability to perform optical real-time spectral analysis of A-scans [16,17]. Also, recently it has been shown that the dispersion properties of the diffraction grating can be exploited by using the relationship between the grating axis and the sample depth [9]. In this way, one can tunably compensate for the dispersion imbalance between the two arms of the interferometer, but the decoupling of the second and third order dispersion terms, will not be possible.

Time domain scan-free optical correlation techniques encompass many configurations that can be found in literature. A fibre system has been already proposed in 2006 [18] following works of Hauger in
2003 [13] and coupled to that of Zeylikovich in 1998 [19]. The general principle of these techniques is similar to the one depicted in Fig. 2. For simplicity, in this figure we focus on the recombination of light from the reference and sample arms, where the temporal correlation takes place.

The lens L1 images the diffraction grating plane R onto the CCD detector plane D. Two temporal signals $r(t)$ and $s(t)$, that have the same polarization states are incident onto the grating $(R)$ with opposite angles $\theta_{i}$ and $-\theta_{i}$. The Fourier transform of the signals $r(t)$ and $s(t)$, gives the complex spectra $\hat{R}(v)$ and $\hat{S}(v)$, respectively. These can be expressed in their complex form $\hat{R}(v)=R(v) e^{j \cdot \varphi_{R}(v)}$ and $\hat{S}(v)=$ $S(v) e^{j \cdot \varphi_{S}(v)}$. The moduli of these spectra are directly accessible in the spectral plane of the spectrometer, which is physically in the back focal plane of the lens L1.

Light at wavelength $\lambda_{i}$ coming from $r(t)$ and $s(t)$, focuses on to the spectral plane at two points that are symmetrical with respect to the optical axis. In the detector plane $\mathrm{D}$, these points produce a classical two wave interference pattern of period $T_{f}=\frac{\lambda_{i}}{2 \cdot \sin \theta_{d}^{\prime}}$, where $\theta_{d}^{\prime}$ is the diffraction angle at wavelength $\lambda_{i}$. By integrating the individual fringe intensities over the spectral bandwidth of the light source, the signal $C(z)$ in the plane $\mathrm{D}$ of the detector is:

$$
C(z)=I_{0}+2 \mathcal{R} e\left[\int_{v} \hat{R}(v) \hat{S}(v) \cdot e^{-j 2 \pi\left(\frac{2 z}{\gamma c} \sin \theta_{i}\right) v} e^{j \frac{4 \pi z}{\gamma \Lambda}} d v\right]
$$

where $z$ is the horizontal coordinate on the CCD camera lines, $I_{0}$ is the background intensity, $\Lambda$ is the grating periodicity, $\gamma$ is the magnification of the imaging system and $c$ is the speed of light in vacuum. Eq. (1) clearly shows the correlation operating between the temporal fields realised by the system. The temporal variable is spatially displayed through the variable change $t=\frac{2 z \sin \left(\theta_{i}\right)}{c}$.

\subsection{Spatial aberration vs induced dispersion law}

Fig. 3 illustrates the principle behind translating the wavefront aberration into a dispersion compensating mechanism. Fig. 3(A) shows how the two points $\mathbb{A}$ and $\mathbb{B}$ at the grating, are imaged through the lens onto the detector. In the detector plane they correspond to points $\mathbb{A}^{\prime}$ and $\mathbb{B}^{\prime}$, respectively. The presence of the diffraction grating implies that for a given point to be imaged, different $k$ vectors corresponding to different colours, simultaneously merge at the detector as seen in the figure. Here, $\Sigma$ and $\Sigma^{\prime}$ are 'coloured' wavefronts associated to the imaging of points (i.e. surfaces of equal optical paths). In order to understand the key principle, consider an achromatic and stigmatic imaging where no wavefront distortion is present, hence there will be no optical path difference for different wavelengths (or $k$ vectors). Consequently, an incident pulse at point $\mathbb{A}$

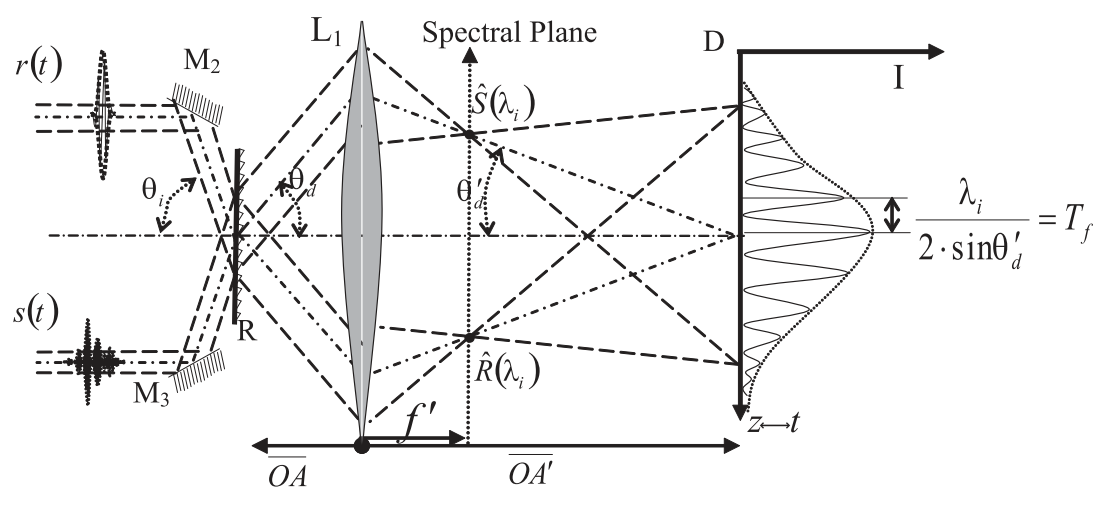

Fig. 2. Working principle of the scan-free correlator. 


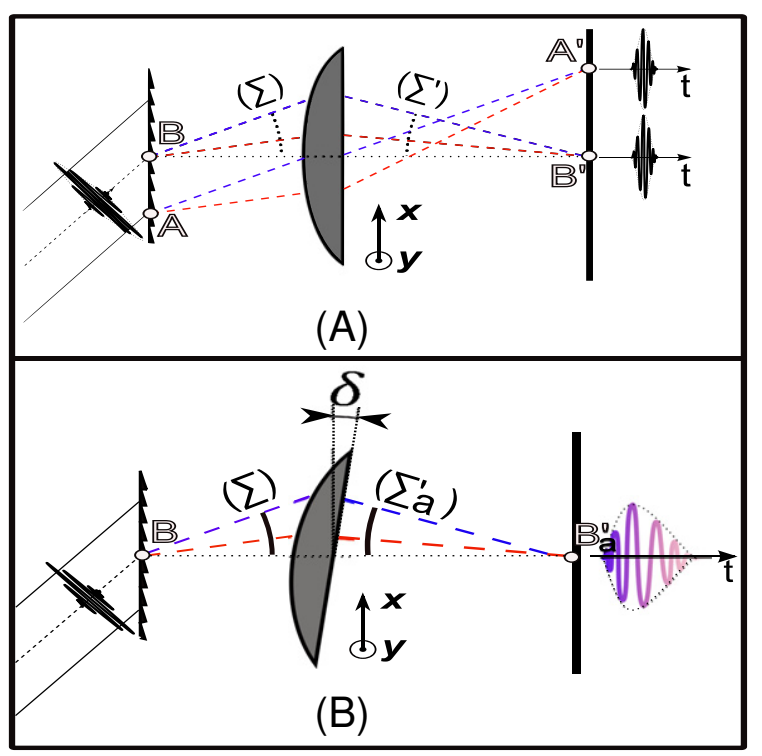

Fig. 3. Aberration effect on the temporal pulse shape. (A) Aberration-free temporal imaging of the pulse that is diffracted by the grating and passes through the lens; $\Sigma^{\prime}$ wavefront is the non aberrated image of $\Sigma$ through the lens. $\mathbb{A}^{\prime}$ and $\mathbb{B}^{\prime}$ are the images of $\mathbb{A}$ and $\mathbb{B}$, respectively, through the lens. (B) Temporal image of a diffracted pulse through the lens when aberration is introduced through tilting of the lens by an angle $\delta$; $\Sigma_{a}^{\prime}$ is the aberrated wavefront when aberration is introduced through the imaging lens.

(or $\mathbb{B}$ ) will not encounter any additional dispersion and this is the reason why pulses at the output points $\mathbb{A}^{\prime}$ and $\mathbb{B}^{\prime}$ are not distorted with respect to the incident one.

Now consider the case depicted in Fig. 3(B) where the imaging lens has been titled by an angle $\delta$. This produces geometrical aberrations like coma or astigmatism. According to wavefront aberration theory, this leads to a deformation of the stigmatic wavefront $\left(\Sigma^{\prime}\right)$ which gives in turn $\left.\Sigma_{a}^{\prime}\right)$. We can express it through a polynomial expansion, using polar coordinates $(\rho, \theta)[20]$ :

$\mathcal{W}\left(x_{0}, \rho, \theta\right)=\sum_{j, m, n} \mathcal{W}_{k l m} x_{0}^{k} \rho^{l} \cos ^{m} \theta$

with $k=2 j+m, l=2 n+m$ and where $\mathcal{W}_{k l m}$ are wavefront aberration coefficients related to the Seidel coefficients. Also $x_{0}$ is the normalised pupil coordinate. We can express the coma wavefront aberration as:

$\Delta \mathcal{W}_{\text {coma }}=\mathcal{W}_{131} x_{0} \rho^{3} \cos \theta$

where $\mathcal{W}_{131}$ is the coma aberration coefficient. In pupil coordinates $(x, y)$ the expression becomes:

$\Delta \mathcal{W}_{\text {coma }}=\mathcal{W}_{131} x_{0} x\left(x^{2}+y^{2}\right)$

There is clearly a third order coefficient term with $x$. This is linked to the wavelength through the diffraction grating effect. As a result, the pulse experiences a third order induced delay term. Consequently, optical paths for the different wavelengths incident onto $\mathbb{B}_{a}^{\prime}$ experience a time delay that is directly proportional to these coefficients. Hence, in Fig. 3(B) the output pulse at point $\mathbb{B}_{a}^{\prime}$ is dispersed.

We also need to encompass the astigmatism induced from tilting into our theory. Astigmatism is expressed in pupil coordinates as:

$\Delta \mathcal{W}_{\text {ast }}=\mathcal{W}_{222} x_{0}^{2} x^{2}$

Essentially, this a second order term which can be compensated by the dual fibre stretchers, as explained earlier. Also, for our case, the effects of other aberrations due to the points being imaged outside of the optical axis, is negligible, as the image area is small compared to the magnification.

\section{Results}

In this section we shall first describe the various components of the setup shown in Fig. 1, after which we shall present and discuss our results from a qualitative and quantitative perspective. The light source is a supercontinuum source provided by Leukos Innovative optical systems. This gives an output power of $80 \mu \mathrm{W} / \mathrm{nm}$ over a bandwidth of $420 \mathrm{~nm}-2400 \mathrm{~nm}$ with a repetition rate of $20 \mathrm{kHz}$. We filter the source using a grating and a spatial filter to obtain a $165 \mathrm{~nm}$ bandwidth centered at $688 \mathrm{~nm}$, which is optimal for our detection system. This spectral bandwidth is equivalent to a temporal pointspread function (PSF) that has a full-width at half-maximum (FWHM) of $2.1 \mu \mathrm{m}$ as seen in Fig. 4. The light from the source is split at the input with a 90/10 coupler to maximise the light incident onto the sample. This arm incorporates a 50/50 coupler which directs the light towards the sample and collects the reflected signal. The PSF of the system is observed when the sample is replaced by a mirror. Both the fibre stretchers in the setup are made up of $4 \mathrm{~m}$ of fibre which are used for dispersion and path length tunability (for more details refer to [8]). The stretcher in the sample arm and most of the Mach-Zehnder interferometer, including the couplers, is made up of FiberCore SM800 fibre. For that fibre we measured a dispersion coefficient, $\beta_{2}^{A} \simeq$ $47 \mathrm{ps}^{2} / \mathrm{km}$ at $700 \mathrm{~nm}$. On the other hand, for the reference arm stretcher, we used some Crystal Fibre LMA-5 photonic crystal fibre (PCF). This fibre possesses properties similar to that of the SM800 fibre in terms of transparency and core diameter, while simultaneously exhibiting a different dispersion parameter, $\beta_{2}^{B} \simeq 37 \mathrm{ps}^{2} / \mathrm{km}$ at $700 \mathrm{~nm}$. Note that both types of fibres are present in each arm of the interferometer as explained in [8]. The polarisation controllers at the input and in the reference arm ensure that the polarisation state of the light is aligned over the entire spectrum, thus providing optimum fringe contrast. Light exiting the fibre output from each of the interferometer arms, is collimated with a microscope objective (Leica Achr $10 \times$ infinity corrected) and passes through a $600 \mathrm{~g} / \mathrm{mm}$ transmission diffraction grating, optimised for $600 \mathrm{~nm}$ from Wasatch Photonics. The imaging lens is an achromatic lens from Newport (PAC070) and the detector is an Ueye CCD detector with $1024 * 1280$ pixels (UI-2240SE-M).

\subsection{Experimental results and qualitative analysis}

Without any dispersion compensation, we observe the experimental normalised PSF shown in Fig. 5. The FWHM is degraded to

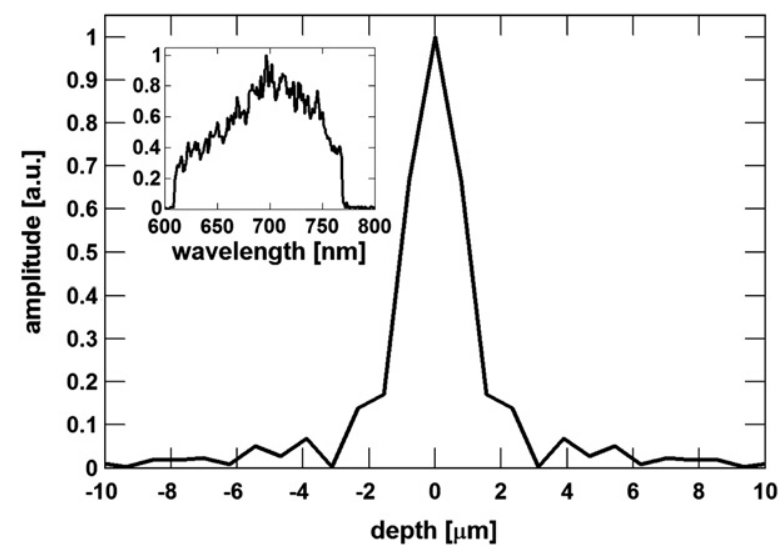

Fig. 4. Spectrum of the light source and temporal PSF. The spectrum can be seen in the inset and the corresponding normalised theoretical PSF is in the main window. 


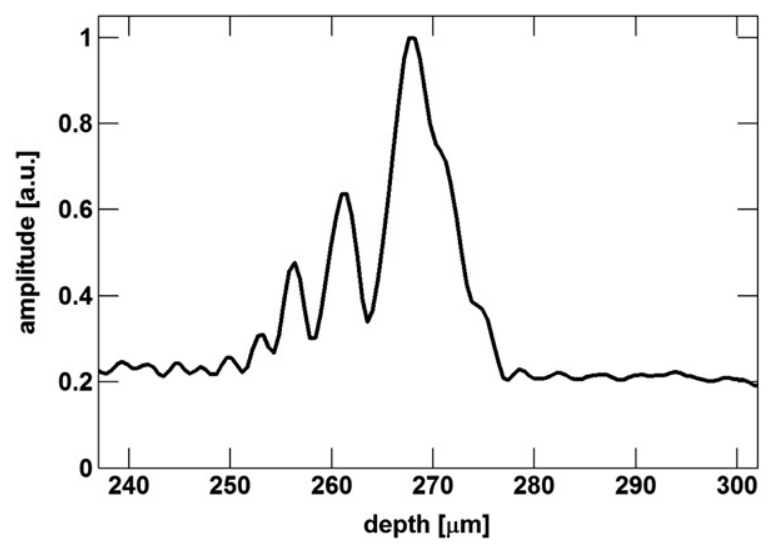

Fig. 5. Normalised temporal PSF when the dispersion is imbalanced.

$12 \mu \mathrm{m}$, which is nearly 6 times broader than the diffraction limited one. The shape of the PSF exhibits both 2nd and 3rd order dispersion terms as well as higher order terms. We proceed by compensating for the 2nd order dispersion term through the dual stretchers $\left(95^{\circ}\right.$ rotation of the PCF stretcher corresponds to a $2.4 \mathrm{~mm}$ elongation of the PCF) which results in a measured PSF of about $5.1 \mu \mathrm{m}$ that can be seen in Fig. 6. When we take a closer look, we observe some residual higher order terms that cannot be further compensated by the stretchers alone. The next step was to tilt the imaging lens by an angle $\delta=3.4^{\circ}$. This angle has been chosen experimentally by looking at the shape of the PSF. When the PSF is symmetrical and as sharp as possible then the tilt angle is registered. This dramatically decreased the FWHM of the temporal PSF down to $2.8 \mu \mathrm{m}$ as seen in Fig. 7. However, there are still some remaining higher order dispersion terms that could neither be suppressed by the stretchers, nor the lens. Nonetheless, the obtained PSF is very close to the diffraction limited one. When looking carefully at the Fig. 7 we may notice a very small decreasing of the PSF contrast with respect to Fig. 6. From a principle point of view, there is no reason why the PSF should decrease due to dispersion compensation. In fact, when we performed several experiments with the lens (see ref [5]), we noticed an increase of the PSF amplitude with dispersion, as expected. We believe that the small decrease in PSF contrast is due to a small 'undesired' defocus added to the tilt of the lens, which decreases the incident power on the camera, hence reducing the SNR. In the next section we shall quantitatively analyze these results and compare it to the theoretical predictions.

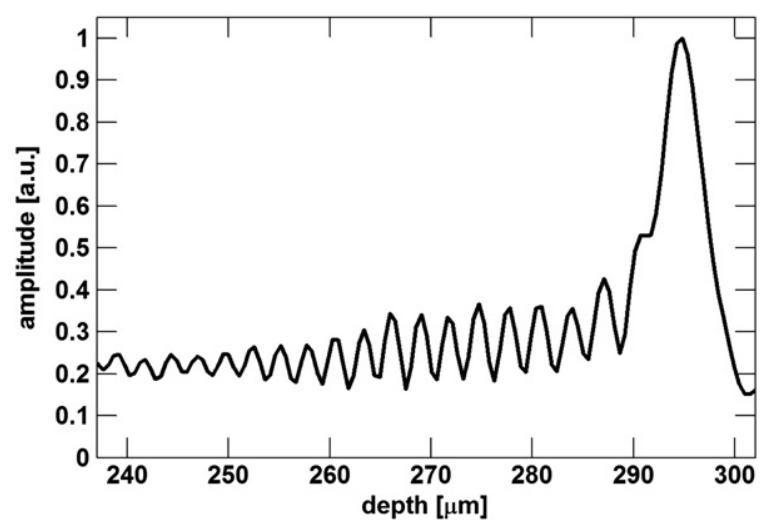

Fig. 6. Normalised temporal PSF when the dispersion is compensated by only the stretchers.

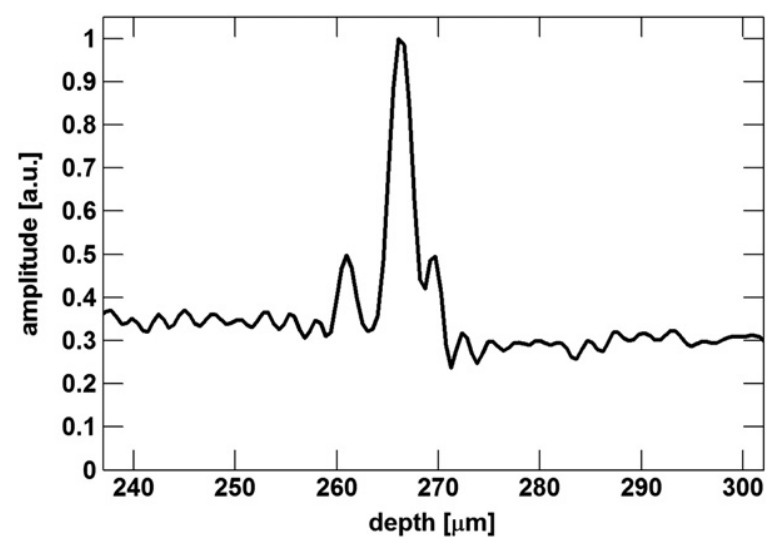

Fig. 7. Normalised temporal PSF when the dispersion is compensated by the stretchers and tilting the lens.

\subsection{Quantitative analysis}

\subsubsection{Phase analysis}

Fig. 8 shows the calculated experimental Optical Path Difference (OPD) (the red OPD $=\frac{\text { phase } \cdot \lambda}{2 \pi}$ ) for the three PSFs in the following cases :

- No dispersion compensation

- Dispersion compensation through only the stretchers

- Lens dispersion compensation added to the stretchers

In all the graphs the linear term of the power series expansion as well as the constant one have been removed. Fig. 8(A) is the optical path difference (OPD) extracted from the experimental PSF for the three previously mentioned cases. We fitted the experimental data with a 4th degree polynomial. The vertical axis is the OPD expressed in terms of the number of wavelengths $(\lambda)$ with respect to the central wavelength of the source. Although Fig. $8(B)$ is very similar to the previous graph, here we only plot the fitting curves and limit the fit to the 2nd and 3rd order. By closely observing both these graphs, it is clear that the stretcher mainly compensates for the 2nd order dispersion. The resulting effect is that the phase after stretcher compensation is almost flat between $3.9 \times 10^{14} \mathrm{~Hz}$ and $4.5 \times 10^{14} \mathrm{~Hz}$ and leads to an equivalent theoretical PSF FWHM of about $6 \mu \mathrm{m}$ which is consistent with the measured one. Beyond $4.5 \times 10^{14} \mathrm{~Hz}$ we notice a strong decrease in the OPD for the stretcher curve which almost reaches $-4 \lambda$. The energy in the higher frequencies is also decreasing in this wavelength range (see the inset of Fig. 4). This is the reason why we see an asymmetric spreading of the PSF (Fig. 6) for high frequencies, but with an amplitude lower than that of the main peak.

The lens effect can be clearly understood as a third order correction term as seen in Fig. 8(A) and (B). In Fig. 8(B), upon first glance it may seem that the lens is increasing the OPD. However, while it does increase the second order term, a fourth order term is also growing in the opposite direction, leading to a global flattening that can clearly be seen in Fig. 8(A). Finally, on the same figure we see that the residual higher order terms (even and odd ones) are still present, but are low enough $(\leq 0.2 \lambda)$ to give a global PSF close to the diffraction limited case.

\subsubsection{Comparison with theory}

3.2.2.1. Stretcher compensation. The effect of optical fibre stretching on dispersion coefficients has been widely studied for over two decades $[21,23]$. Recently, its application to OCT was demonstrated by Iyer et al. about a year ago [8]. Basically, it was shown that the stretch 

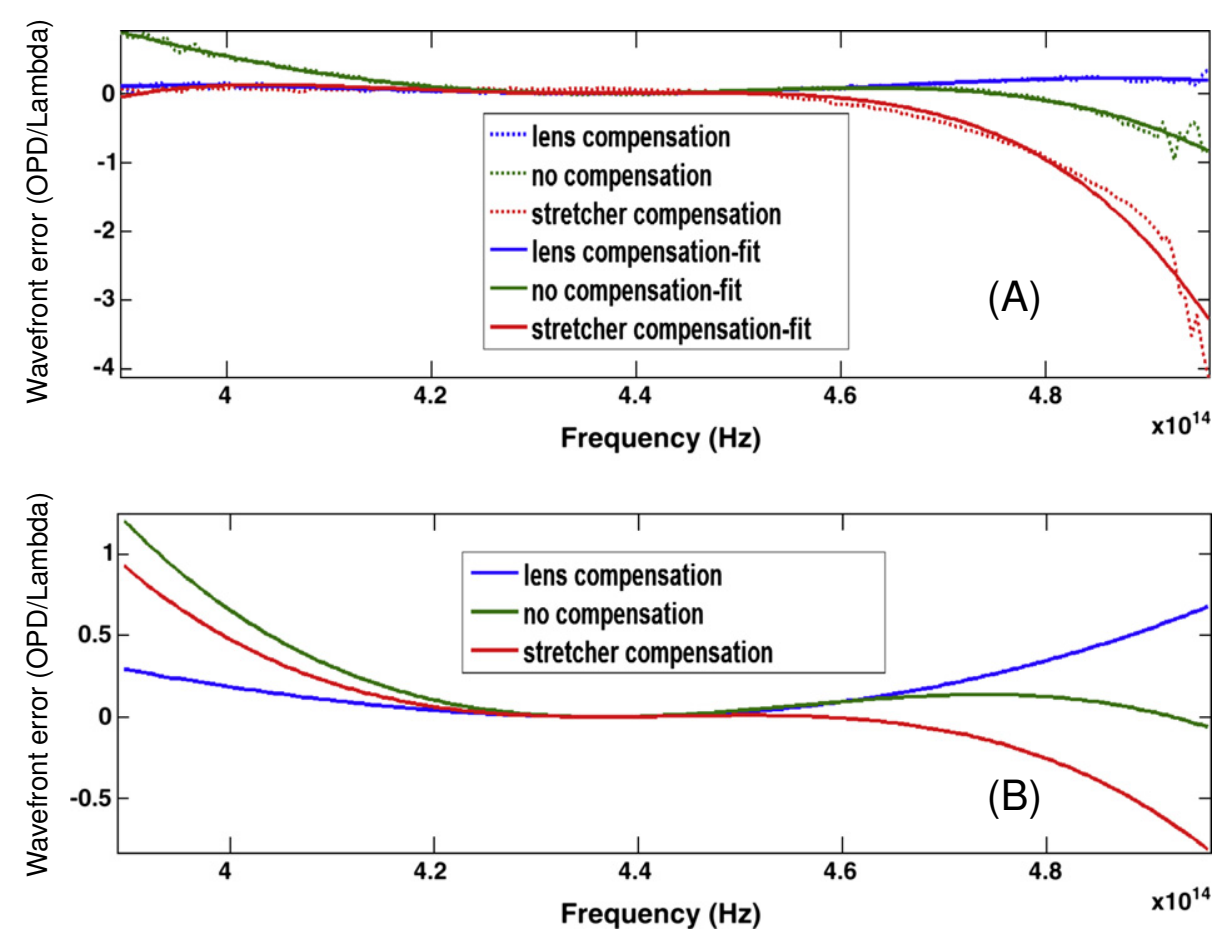

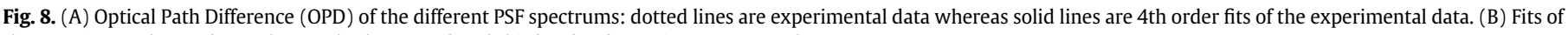
the experimental OPD data, where only the second and third order dispersion terms are shown.

induced phase change developed up to the 3rd order can be written as:

$$
\begin{aligned}
\Delta \Phi= & \frac{\beta_{2}}{2} \cdot\left(\omega-\omega_{0}\right)^{2} \cdot C_{P C F} \cdot \Delta L \\
& +\frac{\beta_{3}}{6} \cdot\left(\omega-\omega_{0}\right)^{3} \cdot \Delta L
\end{aligned}
$$

where $\beta_{2}$ is the second order dispersion coefficient (we measured $\left.\beta_{2}=37 \mathrm{ps}^{2} / \mathrm{km} @ 700 \mathrm{~nm}\right), \beta_{3}$ is the third order dispersion coefficient (we measured $\beta_{3}=42 \cdot 10^{-14} \mathrm{ps}^{2} / \mathrm{km} @ 700 \mathrm{~nm}$, which is much smaller than $\left.\beta_{2}\right), \omega$ is the light angular frequency, $\omega_{0}$ is the central light angular frequency, $C_{P C F}$ is a coefficient that accounts for the strain-induced optogeometrical changes of the PCF fibre (we measured $C_{P C F}=0.45$ ) and $\Delta L$ is the change in fibre length after stretching $(\Delta L=2.4 \mathrm{~mm}$ in this experiment). In principle, there should also be a coefficient for straininduced optogeometrical changes acting on $\beta_{3}$, however, as $\beta_{3}$ is much smaller than $\beta_{2}$, this term is not significant. Hence, we do not account for this coefficient in the following analysis and this is partially the reason for the mismatch between the theoretical and experimental values.

In Fig. 9, there is excellent agreement between the theoretical stretcher compensation based on a third order development and the experimental curve. The nearly constant residual 4\% error is mainly due to the limited development of the theoretical dispersion law, and partially due to the difference that exists between the effective $\beta$ coefficients and the computed ones.
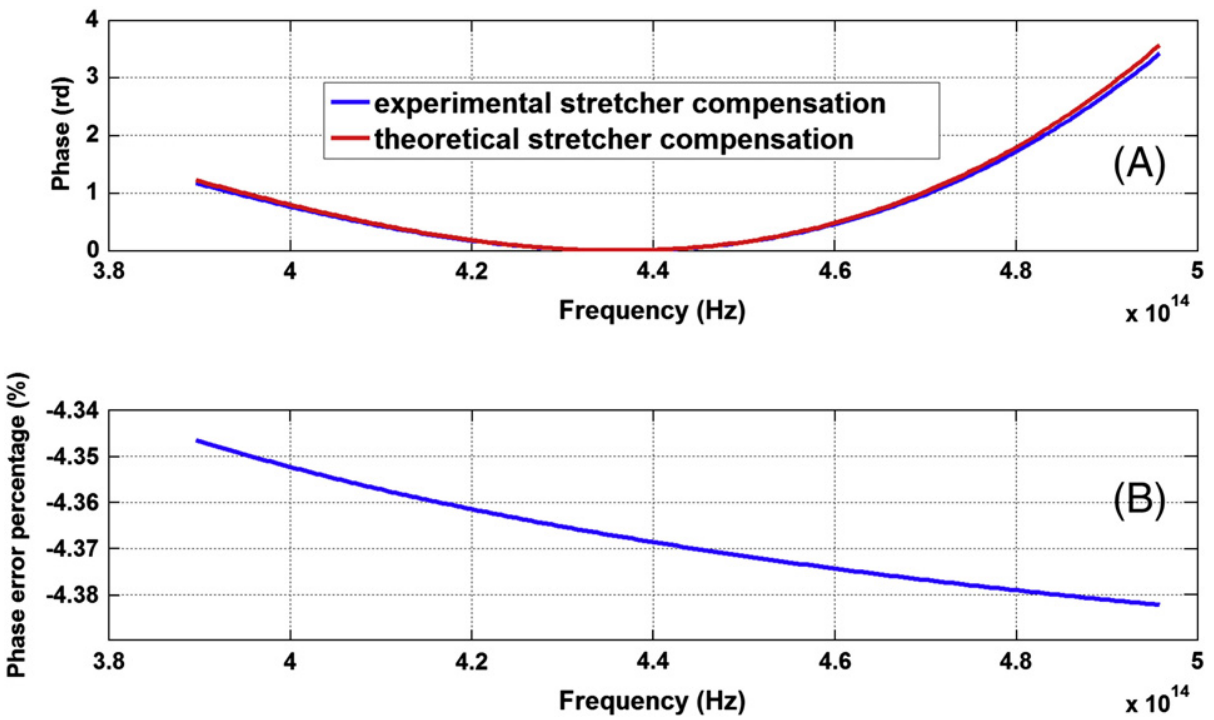

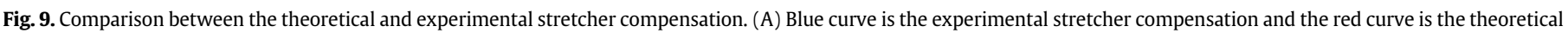
one. (B) Difference between theory and experiment (expressed in percentage). 
3.2.2.2. Lens compensation. As explained in Section 2.3, tilting of the lens induces a coma aberration which is a third order term with respect to the angle, and in turn to the wavelength. This aberration is then directly a third order dispersion law. A simulation of the aberration induced by tilting of the lens by an angle $\delta=3.4^{\circ}$, was done in ZEMAX and the obtained OPD was converted into a dispersion law using Matlab. The resulting temporal PSF is shown in Fig. 10. There is very good agreement between the experimental result shown in Fig. 10(b) and the theoretical simulation shown in Fig. 10(c). We see some residual mismatch on the left side of the PSF which could be due to several reasons: a discrepancy in the centering on the lens or a variation of the lens in manufacturing to specifications given in the data sheet. Despite this small error, it is clear from Fig. 10 that both the experimental and theoretical PSFs are close to the diffraction limited case given in Fig. 10(a).

\subsubsection{Discussion}

3.2.3.1. Stretcher. We have shown that the stretchers can efficiently compensate for the first and second order dispersion terms without significantly altering the third order. However, the limitation of our stretchers is the maximum dispersion mismatch that can be induced by stretching the optical fibres. This is dependent on two main parameters:

- The difference in the dispersion coefficients of the two fibres in each arm of the interferometer.

- The maximum length of fibre that can be elastically stretched by the rubber stretchers.

The first parameter is subject to future work as there is a growing effort to create fibres with strong variations in their composition and/ or structure [22]. These changes in turn affect the dispersion coefficients and their behaviour with respect to the wavelength. This is certainly a feasible way to achieve higher dispersion compensation and also to change the ratio between the second and third order dispersion coefficients.

The second parameter is governed by the Young's modulus of the fibre. But, silica fibres can only be stretched by a maximum of 2\%[23]. So, an alternative approach to maximise the dispersion mismatch would be to increase the length of fibre wrapped around the stretch- ers, but when using PCF fibres this option may become expensive. Nonetheless, the main advantage of the dual stretchers is the ease of implementation and compactness due to its all-fibre format.

3.2.3.2. Lens compensation. As we have demonstrated earlier, lens compensation can correct for third order dispersion. In fact we have seen that the degree of dispersion compensation we add with the lens is directly dependent on the spatial aberration we induce with the lens itself. Generally, in an imaging system, adding spatial aberration decreases its spatial resolution. In Fig. 11, lens (L) is forming an image of the grating $(G)$ onto the detector (D). Here, spatial aberrations should decrease the lateral resolution on the detector (D). As the lateral axis on the detector is connected to the depth into the sample (dimension $z$ ) we may infer that the spatial aberration cancels the dispersion compensation due to the lens coma. However, this is not the case as was demonstrated from the previous experiments. This apriori paradox can be understood from Fig. 11. Let $\mathcal{R}_{B}$ and $\mathcal{S}_{B}$ be the light exiting the reference and sample arms, respectively. The temporal signals carrying the tomographic information lies within the entire beam profile (i.e. there is no spatial localization into the sample beam of the tomographic information). $\mathcal{R}_{B}$ is transmitted through the zeroth order of the diffraction grating $(G)$, whereas $\mathcal{S}_{B}$ is diffracted through the -1 order. The lens $(L)$ images $(G)$ on plane $(I)$ so that $\mathbb{A}$ is imaged on $\mathbb{A}^{\prime}$ and $\mathbb{B}$ on $\mathbb{B}^{\prime}$. In the presence of aberration (we have chosen defocus, for the sake of clarity in the diagram) the detector plane (D) no longer matches the image plane (I). The only region in the detector plane (D) where all the spectral content from all the beams is attained, lies in the region between a and $\mathbf{b}$. Here, all the beams that are overlapping can be seen in the Fig. 11. On one hand as between $\mathbf{a}$ and $\mathbf{b}$ all beams wavelength content is present the Fourier transform gives the complete information about temporal fields, hence there is no information loss in the correlation between those temporal fields. On the other hand as the overlapping field is limited in the detector plane it is also limited in the object plane (i.e. the grating plane $(G)$ ). By back propagation through the lens $(\mathrm{L})$ of the points a and $\mathbf{b}$ which are limiting the field, we find points $\mathbb{A}^{\prime \prime}$ and $\mathbb{B}^{\prime \prime}$ which are limiting the effective OCT system depth field which is imaged through the lens when aberration is induced. To summarise, inducing an aberration effectively compensates for a dispersion order related to the aberration order, but this leads to a decrease in the imaging depth field of the whole OCT System.
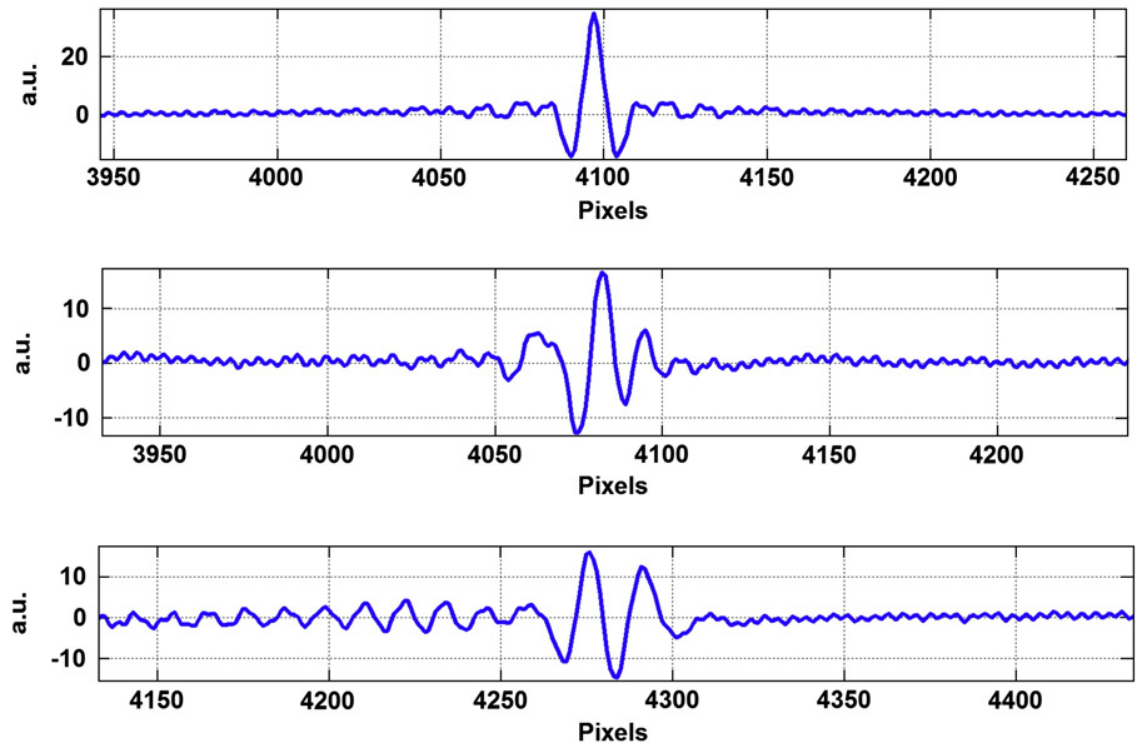

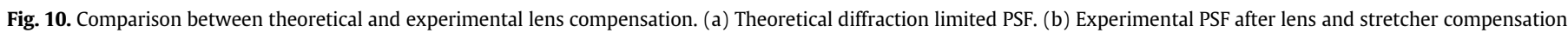
(c) Obtained PSF when theoretical lens compensation is added to the experimental stretcher compensated PSF. 


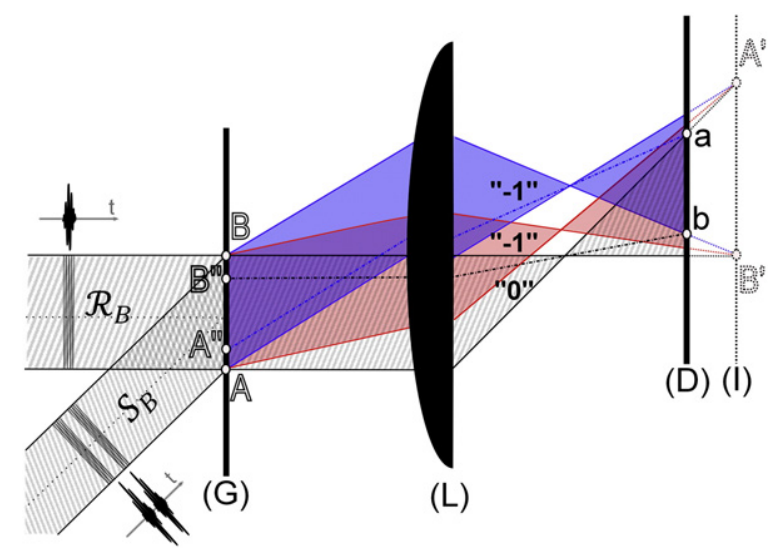

Fig. 11. Effect of spatial aberration on the OCT depth range imaging capability.Here the components are: diffraction grating $(G)$, imaging lens (L), detector plane (D), image plane (I)." 0 " and " -1 " refer to the diffracted order for the shaded colours.

3.2.3.3. Extension of the method to the Near Infra Red range. Most OCT systems opperate in the NIR range. The reason why we didn't realize our experiment in the classical $840 \mathrm{~nm}$ or $1300 \mathrm{~nm}$ central wavelengths is because we combined two independent setups designed for different applications and both of them were not optimized to work together (the cutoff wavelength of the fibre system used in the setup was at $700 \mathrm{~nm}$ and they were optimized to work around $830 \mathrm{~nm}$ ). As we intended to perform our proof-of-principle experiment with the largest source bandwidth (greater than $150 \mathrm{~nm}$ ), we were facing the following dilemma with the fibred setup. If we move towards the longer wavelengths (i.e. greater than $850 \mathrm{~nm}$ ), it does work as we utilized $800-880 \mathrm{~nm}$ in the experiment in Ref. 8. But, since we are using a much larger bandwidth, the bend loss becomes a lot more significant especially with the use of stretchers that accentuate the sharp loss between the shorter and longer wavelengths. Now, if part of our wavelength window is below the cutoff wavelength then we would be in the multimode regime. But, since we were only using short lengths of fibre $(\sim 7 \mathrm{~m})$, this didn't appear as a problem to us. So, with a different fibre that is less susceptible to bend loss around $(850 \mathrm{~nm})$, we can demonstrate broad bandwidth 2nd order compensation with the setup. Nonetheless, for the proof-of-principle what we have demonstrated is adequate, and could be extended to the NIR range with adequate fibres. In terms of the scan-free system, in Ref. 5 we showed that lens compensation becomes more efficient in the long wavelength range. We have performed experiments for the $800-900 \mathrm{~nm}$ bandwidth that showed successful independent removal of the second and the third order term of dispersion. However to maintain a uniform contrast for the entire bandwidth of the experiment, we had to work at shorter wavelength when using almost $200 \mathrm{~nm}$ bandwidth.

\section{Conclusion}

Dispersion compensation in OCT is crucial especially, when dealing with high resolution systems (few $\mu$ m resolution) and/or thicker samples to be observed. Although there are efficient methods to compensate for dispersion in bulk OCT systems, the problem is still relatively complex with fibred systems, as fibres are highly sensitive to any environmental change (pressure, temperature, etc.). Generally, a continual steady variation of the dispersion compensation would be necessary with time to continuously optimise the resolution. The standard way to proceed with any fibred interferometer is to insulate the whole setup as much as possible from external changes. We demonstrated in the frame of this paper that the use of fibre stretchers coupled to a scan-free correlation system enables a tunable and nearly independent dispersion compensation of the 2 nd and $3 r d$ order. The former term is dealt with the dual stretchers that enable tuning of the optical path difference between the two arms of the interferometer, while simultaneously compensating for the 2nd order dispersion. The latter term is dealt with the time domain correlator, which has the property to convert Seidel aberration coefficients into the same order dispersion coefficients, allowing us to compensate for the 3rd order dispersion. Although essentially dedicated to system dispersion compensation, in the frame of this 'proof of principle' paper, we would like to outline potential interests of such a system for a more general purpose. The main advantage of the solution we propose is to be able to independently compensate 2 nd and 3rd order terms which are the main predominant terms involved in OCT. Taking a closer look at the properties of such a system, we hypothesise that average dispersion could also be compensated. Further, we imagine that the tunability of this method will allow us to compensate for depthdependent dispersion in the sample, in a continuous manner. This could be a kind of adjustable depth dependant dispersion compensation if coupled to the fact that real time A-scans (or B-scans; see ref [16]) are obtained with scan free systems. At this point, we would like to make a few remarks about the system:

- Scan-free time domain detection systems share, in principle, the same sensitivity limitations with TDOCT. Clearly, this limitation is critical when both sensitivity and real-time conditions are simultaneously needed. Nevertheless, authors have recently shown high speed imaging capability together with relatively good sensitivity in in-vivo biological media $[24,25]$.

- Specific properties of these systems, such as dispersion compensation, optical processing of Wigner functions, potentially depth dependant dispersion compensation and artefact-free optically processed time domain imaging, are highly attractive and unique features [12]. Such systems may also become a standard for functional imaging in some media where real-time access to functional information is more important than the sensitivity (i.e. less scattering samples and/or fewer constraints in incident light power). The complex A-scan is directly obtained without scanning, or any numerical signal processing in the detector plane. Having said that, the demodulation of the A-scan and noise filtering, require numerical processing. When we speak about 'post-processing' free, this means without demodulation. Then the 'post-processing' free property (as compared to FDOCT) and scan-free (as compared to TDOCT) becomes a key factor, especially in the frame of spectroscopic OCT.

- Finally, this detection modality is a free-space scheme making it a hybrid system by combining fibre and free-space components. For endoscopic type measurements, the fibred imaging part could be separated from the free-space one, outside the region of interest.

To have a complete overview of the impact of such a hybrid system in the field of in-situ biological imaging, the complexity of the system with respect to obtained results needs to be addressed. Indeed, actual fibred OCT systems are still less developed than external systems, often due to the complexity of the system. Scan-free systems, with of course an inherent lower sensitivity, show globally simpler architectures and may, in the frame presented earlier, be a good candidate for fibre systems.

\section{Acknowledgments}

Frederique Vanholsbeeck acknowledges the support from the research and study leave funding from the University of Auckland.

Luc Froehly thanks the ANR which has been funding part of this project during 2005-2008.

\section{References}

[1] D. Huang, E.A. Swanson, C.P. Lin, et al., Science 254 (1991) 1178.

[2] M.T. Tsai, I.J. Hsu, C.W. Lu, Y.M. Wang, et al., Opt. Quantum Electron. 37 (2005) 1199.

[3] T. Hillman, D. Sampson, Opt. Express 13 (6) (2005) 1860. 
[4] E.D.J. Smith, A.V. Zvyagin, D.D. Sampson, Opt. Lett. 27 (22) (2002) 1998

[5] B. Cense, N.A. Nassif, T.C. Chen, M.C. Pierce, S.H. Yun, B.H. Park, B.E. Bouma, G.J Tearney, J.F. de Boer, Opt. Express 12 (11) (2004) 2435

[6] M. Wojtkowski, V. Srinivasan, T. Ko, J. Fujimoto, A. Kowalczyk, J. Duker, Opt. Express 12 (11) (2004) 2404.

[7] Y. Chen, X. Li, Opt. Express 12 (24) (2004) 5968.

[8] S. Iyer, S. Coen, F. Vanholsbeeck, Opt. Lett. 34 (2009) 2903.

[9] L. Froehly, L. Furfaro, P. Sandoz, P. Jeanningros, Opt. Commun. 282 (2009) 1488

[10] P. Connes, J. Mod. Opt. 4 (1957) 136

[11] R. Trebino, Frequency-resolved optical gating: the measurement of ultrashort laser pulses, Kluwer Academic Publishers, 2002.

[12] L. Froehly, R. Leitgeb, J. Opt. 12 (2010) 084001.

[13] M.W.C. Hauger, T. Hellmuth, Appl. Opt. 42 (2003) 3896.

[14] W. Drexler, J.G. Fujimoto, Optical coherence tomography: technology and applications, Springer Verlag, 2008.

[15] Y. Watanabe, F. Sajima, T. Itagaki, K. Watanabe, Y. Shuto, Appl. Opt. 48 (2009) 3401.
[16] L. Froehly, M. Ouadour, G. Petitjean, L. Furfaro, P. Sandoz, T. Gharbi, P. Leproux, G. Huss, V. Couderc, Real-time optical coherence spectrotomography: proof of principle, volume 6191 of Biophotonics and New Therapy Frontiers SPIE, Apr. 2006.

[17] L. Froehly, M. Ouadour, L. Furfaro, P. Sandoz, T. Gharbi, P. Leproux, G. Huss, V. Couderc, Spectroscopic OCT by grating-based temporal correlation coupled to optical spectral analysis, International Journalof Biomedical Imaging, 2008, 2008.

[18] P. Koch, V. Hellemanns, G. Hüttmann, Opt. Lett. 31 (2006) 2882.

[19] I. Zeylikovich, A. Gilerson, R.R. Alfano, Opt. Lett. 23 (1998) 1797.

[20] J.C. Wyant, K. Creath, Robert R. Shannon, in: Robert R. Shannon, James C. Wyant (Eds.), Applied Optics and Optical Engineering, vol. XI (11), Academic Press, Inc. New York, 1992, p. 2.

[21] D.A. Jackson, R. Priest, A. Dandridge, A.B. Tveten, Appl. Opt. 19 (1980) 2926

[22] W.H. Reeves, J.C. Knight, P.St.J. Russell, P.J. Roberts, Opt. Express 10 (2002) 609.

[23] L. Simohamed, F. Reynaud, Opt. Commun. 159 (1999) 118.

[24] Y. Watanabe, K. Yamada, M. Sato, Opt. Express 14 (2006) 5201

[25] Y. Watanabe, F. Sajima, T. Itagaki, K. Watanabe, Y. Shuto, Appl. Opt. 48 (2009) 3401. 